\title{
Cobalt Ferrite Nanoparticles from Single and Multi- Component Precursor Systems
}

\begin{tabular}{|r|l|}
\hline Journal: & Zeitschrift für Anorganische und Allgemeine Chemie \\
\hline Manuscript ID: & zaac. 200900010 \\
\hline Wiley - Manuscript type: & Article \\
\hline Date Submitted by the & 07-Jan-2009 \\
\hline Complete List of Authors: & $\begin{array}{l}\text { Mathur, Sanjay; Institute of Inorganic Chemistry } \\
\text { Cavelius, Christian; Leibniz Institute of New Materials } \\
\text { Moh, Karsten; Leibniz Institute of New Materials } \\
\text { Shen, Hao; Department of Inorganic and Materials Chemistry } \\
\text { Bauer, Jürgen; Leibniz Institute of New Materials }\end{array}$ \\
\hline Keywords: & Cobalt Ferrite, Single-Source Precursor \\
\hline &
\end{tabular}

\section{S ScholaroNE \\ Manuscript Central}


1

2

3

4

5

6

7

8

9

10

11

12

13

14

15

16

17

18

19

Dedicated to Prof. Dr. Gerd Meyer as a tribute to his seminal contributions to solid-state and coordination chemistry

*Corresponding author: sanjay.mathur@uni-koeln.de 
Figure for table of contents

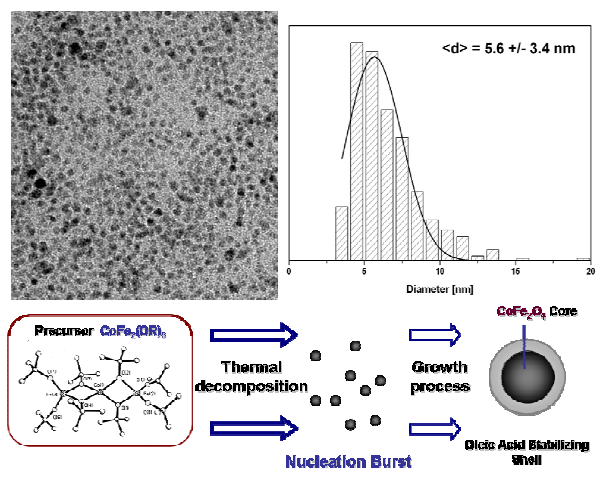




\begin{abstract}
Highly crystalline $\mathrm{CoFe}_{2} \mathrm{O}_{4}$ nanoparticles $(<\mathrm{d}>\sim 5-6 \mathrm{~nm})$ were synthesized by thermal decomposition of (i) single molecular precursor $\mathrm{CoFe}_{2}(\mathrm{OtBu})_{8}$ and (ii) stoichiometric mixture of individual metal precursors. The precursors were thermolyzed in a high-boiling solvent (Docosane, bp. $369^{\circ} \mathrm{C}$ ) to produce uniformly dispersed cobalt ferrite nanoparticles in the case of molecular precursor, whereas bimodal distribution of nanoparticles was obtained in the multi-source synthesis. TG/DSC and HR-TEM reveraled single and multi-step decomposition profiles and differential nucleation steps in the two cases, which influence size dispersion and phase purity.
\end{abstract}

Keywords: Cobalt Ferrite, Thermal Decomposition, Single-Source Precursor

\title{
Introduction
}

Oxidic magnetic nanocrystals like magnetite, maghemite and cobalt ferrite with superparamagnetic properties have been synthesized by different techniques due to its outstanding and tunable magnetic properties ${ }^{1}$. A wide range of applications basing on iron oxide spinells could be addressed like the use in clinical chemistry ${ }^{2}$ as well as ferrofluid for sealing purposes ${ }^{3}$. Beside the essential requirements the greatest challenge for the production of high quality iron oxide systems is the need of a precise control over size, shape and crystallinity because these morphological characteristics allow the engineering of the magnetic properties ${ }^{4}$. Commonly, solution phase methods like the (co)precipitation of the metal ions $\mathrm{M}^{2+}$ and $\mathrm{Fe}^{3+}$ in aqueous media by a base or aerosol-mediated techniques are used to produce large quantities of nanoparticles ${ }^{5}$. Even though, these methods are suited for mass production, the controllable synthesis is difficult to achieve and the produced materials are quite often poorly crystalline and polydispersive.

Alternative approaches produce nanoparticles by thermal decomposition of metal organic precursors ${ }^{6}$ like iron pentacarbonyl, iron acetylacetonate or iron carboxylates in a high 
boiling organic solvent resulting in highly monodispersive monometallic nanoparticles ${ }^{7}$. Metal organic alkoxide precursors can decompose at elevated temperatures (around 140 to $340{ }^{\circ} \mathrm{C}$ ) into metal organic fragments or oxide clusters in a suited high boiling solvent ${ }^{8}$. At a distinct temperature the precursor decomposes homogeneously into the whole solvent. Many seeds are formed during this process growing via cross condensation reactions between the decomposition products which induce a well defined ripening process. This behaviour is used to produce monodispersive crystalline particles with narrow size distribution ${ }^{9}$.

Compared with various other methods like the Sol-Gel-Process it is much easier to produce huge amounts of deagglomerated particles with moderate time and equipment consumption. In general, with a suited heating rate there is no concentration gradient of the metal oxide precursor compound into the solvent, which is a big problem in Sol-GelProcessing of particles including the formation of polydisperse seeds, which is only to avoid by a very precise process control and long / moderate ripening times at soft conditions leading to amorphous particles which has to be crystallized by other methods like sintering or the hydrothermal process. Compared with solvothermal or hydrothermal routes powder production by thermal decomposition is much cheaper because no expensive high-pressure suited equipment is used and by this way nearly no limitations of up-scaling are given. Crystallinity, shape and size of the particles can be regulated by temperature and the in situ capping with stabilizer molecules (mostly long-chain organic mono- or multifunctional molecules), which enables to produce stable colloids with defined properties ${ }^{10}$. Bimetallic precursors can be used to form complex metal oxides with a one-step strategy by a very easy experimental procedure.

Nevertheless, removal of the high boiling organic solvent, which is required to obtain a nanopowder, still remains a challenge. Additionally, the synthesis of particles with bimetallic oxide phases like $\mathrm{CoFe}_{2} \mathrm{O}_{4}$ is difficult due to the different thermal stability of the organic precursors in a mulitcomponent system. Molecular precursors containing an 
appropriate stoichiometry of the required metal ions can be decomposed in a solvent to achieve monodisperse nanoparticles.

In order to control the synthesis of metal oxide nanoparticles, it is important to sperate nucleation and growth phases, which can be ideally achieved by decomposition of metalorganic precursors. When the thermal energy available in the reaction medium (solvent) exceeds the chemical bond energy, an abrupt decomposition of the precursor molecules and a single burst of nucleation occurs producing nuclei of similar sizes. By injecting metal-organic precursors in high boiling solvents $\left(\mathrm{T}>\mathrm{T}_{\text {decomposition }}\right)$ it is possible to achieve the nucleation of particles in a very short time period, which allows the production of $\mathrm{CoFe}_{2} \mathrm{O}_{4}$ nanoparticles with narrow size distribution (Scheme 1).

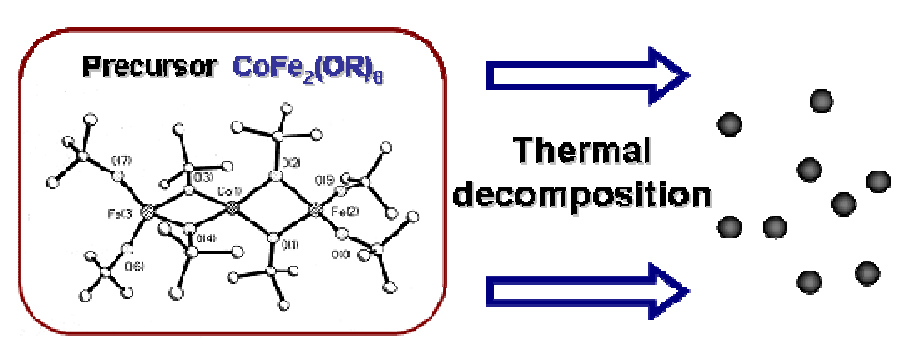

Nucleation Burst



Shell

Scheme 1. Decomposition of precursor molecules in a high boiling solvent and nucleation and growth steps.

In this paper, we present the synthesis of highly crystalline ferrite powders which is based on the thermal decomposition of a single-source precursor $\mathrm{CoFe}_{2}\left(\mathrm{O}^{t} \mathrm{Bu}\right)_{8}$, which contains the elements ideal $\mathrm{Co}$ :Fe ratio for the formation of $\mathrm{CoFe}_{2} \mathrm{O}_{4}$. The surface of the particles is in-situ capped by oleic acid as long-chain surfactant to keep the particle stable and suspended.

\section{Experimental}

Preparation of $\mathrm{CoFe}_{2}\left(\mathrm{O}^{t} \mathrm{Bu}\right)_{8}$ single-source precursor

The preparation of $\mathrm{CoFe}_{2}\left(\mathrm{O}^{\mathrm{t}} \mathrm{Bu}\right)_{8}$ was achieved by salt elimination of sodium iron t-butoxide analogous to the synthesis of $\mathrm{MgAl}_{2}\left(\mathrm{O}^{t} \mathrm{Bu}\right)_{8}$ which was reported before ${ }^{11}$. All operations were 
performed under nitrogen or argon using a modified Schlenk technique. All solvents were dried via standard methods and stored under sodium wire or molecular sieve.

The precursor was synthesized in a $500 \mathrm{ml}$ round bottom flask equipped with condenser, magnetic stirrer. Typically, $4.44 \mathrm{~g}(6 \mathrm{mmol})$ sodium iron t-butoxide was solved in

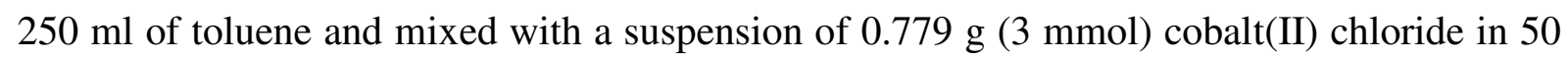
$\mathrm{ml}$ of tetrahydrofurane. After mixing of the two components, the colour turned from greenbrown to deep purple, indicating the formation of the complex. Subsequent heating of the suspension to reflux for $12 \mathrm{~h}$ resulted in the dissolution of the cobalt chloride and the formation of the paramagnetic complex ${ }^{12}$. After filtration of sodium chloride, evaporation of the solvent and sublimation of the crude product at $125^{\circ} \mathrm{C}$ under vacuum $\left(10^{-2} \mathrm{mbar}\right)$ the purified heterobimetallic compound (Fig. 1) was obtained in yields up to $85 \%$.

\section{Preparation of $\mathrm{CoFe}_{2} \mathrm{O}_{4}$ nanocrystals}

In a typical procedure, a mixture of $2.54 \mathrm{~g}(9 \mathrm{mmol})$ oleic acid and $10 \mathrm{~g}$ of docosane is heated to $369{ }^{\circ} \mathrm{C}$ under nitrogen followed by the addition of $755 \mathrm{mg}(1 \mathrm{mmol})$ of $\mathrm{CoFe}_{2}\left(\mathrm{O}^{\mathrm{t}} \mathrm{Bu}\right)_{8}$ dissolved in $2 \mathrm{~g}$ Docosane (at $100{ }^{\circ} \mathrm{C}$ ). Gaseous products indicate the decomposition of the precursor, which is attended by the almost instantly change in colour from purple to black. The solution was kept at $369{ }^{\circ} \mathrm{C}$ for 30 minutes. to complete the decomposition process and then cooled down to $80{ }^{\circ} \mathrm{C}$. Addition of ethanol results in precipitation of the nanocrystals which were purified by standard methods described by Yu et. al. ${ }^{6}$

\section{Instrumentation}

Structure and phase purity of the synthesized nanoparticles were investigated by X-ray

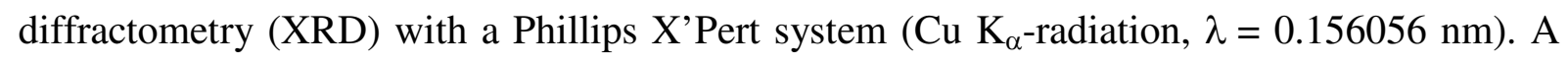
transmission electron microscope (TEM; Philips CM20) was used to examine particle 
crystallinity and morphology. Thermogravimetric analyses (DSC-TG) for precursor decomposition studies were performed under argon at a heating rate of $10 \mathrm{~K} / \mathrm{min}$.

\section{Results and Discussion}

To obtain non-aggregated nanoparticles, the precursor solution is injected into a high boiling solvent and a long-chain carboxylic acid as capping agent according to the procedure recently published by Park $^{13}$ for the preparation of iron oxide nanoparticles. For our purposes we modified the procedure to keep the synthesized Co-Fe alkoxide separated from the capping agent solution to avoid unwanted hydrolysis of the precursor.

After addition of the precursor solution the progress of the reaction can visually be monitored as the colour of the solution changes from dark purple to black accompanied by the formation of gaseous decomposition products. Docosane was chosen due to the fact that it exhibits a very high boiling point of $369^{\circ} \mathrm{C}$, which is necessary to control the decomposition behavior of the precursor. Experiments at lower temperatures with octadecene as high boiling solvent only yield poor crystalline and polydispersive products. For a set temperature, no significant variation of particles size was found by variation of heating rate or concentration and the particle size and shape are mainly determined by the long chain carboxylic acid acting as stabilizer. In any cases, an aging step between 30 and 60 minutes is required to obtain $\mathrm{CoFe}_{2} \mathrm{O}_{4}$ crystalline material in high yields $>80 \%$.

Transmission electron microscopy of $\mathrm{CoFe}_{2} \mathrm{O}_{4}$ nanoparticles reveals the high crystallinity of the as-prepared powder (Fig. 2a). No size-selection process was performed before the measurement. The nanocrystals are of spherical shape with an average diameter of $\sim 5.6 \mathrm{~nm}$ with narrow size distribution. There is no agglomeration of nanoparticles even after several months of storing in toluene or hexane. The particles are separated from each other and can be easily precipitated in polar solvents. High-resolution TEM image (Fig. 2b) reveals the crystalline features of the particles. The lattice fringe $(0.252 \mathrm{~nm})$ can be assigned to $<311>$ 
lattice plane supported by JCPDS file No. [77-0426]. X-ray diffraction was perfomerd for nanoparticles aged at $360{ }^{\circ} \mathrm{C}$ for $2 \mathrm{~h}$. It confirms the spinel structure and no other phases such as cobalt- and iron oxides could be identified. By using Scherrer's formula the average crystallite size was calculated using line broadening of the $<400>,<511>$ and $<440>$ peaks. The average crystallite size $(7.6 \mathrm{~nm})$ determined by XRD pattern is in good agreement with that of nanoparticles $(5.6 \mathrm{~nm})$ estimated from TEM histogram data (Inset, Fig. 2a).

The decomposition behavior of the single-source compound has been studied in comparison to that of the multi-source system, $\mathrm{Co}(\mathrm{acac})_{2}$ and $\mathrm{Fe}(\mathrm{acac})_{3}$. To keep the predefined composition and to transfer the ideal $\mathrm{Co} / \mathrm{Fe}$ ratio at 1:2, a sharp decomposition step of the precursor is required to prevent earlier fragmentation leading to phase impurities and polydisperse phases. Although the pyrolysis of the compound is shifted to higher temperatures one-step decomposition was observed (Fig. 4). After melting of the solid precursor represented by an endothermic DSC-peak at $213{ }^{\circ} \mathrm{C}$ the initial decomposition of $\mathrm{CoFe}_{2}\left(\mathrm{O}^{\mathrm{t}} \mathrm{Bu}\right)_{8}$ onsets at $260{ }^{\circ} \mathrm{C}$ and finishes at $303{ }^{\circ} \mathrm{C}$. At higher temperatures the TG curves show a stable plateau up to the end of the heating programm. For the multi-component system reduced onset temperatures of $\mathrm{Co}(\mathrm{acac})_{2}\left(216{ }^{\circ} \mathrm{C}\right), \quad \mathrm{Fe}(\mathrm{acac})_{3}\left(232{ }^{\circ} \mathrm{C}\right)$ and $\mathrm{Co}(\mathrm{acac})_{2}+$ $2 \mathrm{Fe}(\mathrm{acac})_{3}$ mixture $\left(224^{\circ} \mathrm{C}\right)$ were observed. However, the final temperatures, which indicate the transition to the nano-phase materials are drastically shifted to higher temperatures of 342 and $418.9^{\circ} \mathrm{C}$ respictively. Based on these results, the single-source compound seems to be better suited for particle synthesis in terms of thermal decomposition since the temperature range is quite small whereas two-stage decomposition of the multi-source system was observed. This can be explained due to the rather slow degradation of intermediates, especially in the case of the $\mathrm{Co}(\mathrm{acac})_{2}$ precursor. Transmission electron image (Fig. 5) of nanoparticles obtained from multi-source precursors confirms the two-stage decomposition behaviour resulting in a bimodal size distribution $\left(<\mathrm{d}_{1}>\sim 6.1 \mathrm{~nm} ;<\mathrm{d}_{2}>\sim 21.3 \mathrm{~nm}\right)$. 
The intrinsic strength of chemistry to decipher how simple atomic interactions give rise to complexity can be used to control the arrangement of atoms at multiple length scales, ranging from molecular to extended solid-state structures. The idea behind the chemical synthesis of nanomaterials is to transform molecular precursors into materials under retention of the structural units, which are inherent to the precursor molecule and an integral part of the solid-state structure ${ }^{14}$. The chemical techniques use direct, often very simple, chemical reactions in solid, liquid or gaseous state to produce the solid of interest. For instance, solidstate chemical reactions require grinding, mixing, and subsequent heat-treatments to facilitate diffusion of atoms or ions to form the final product, which depends on the temperature of the reaction and the transport across grain boundaries. Compared to diffusion driven multicomponent synthesis, characterized by individual decomposition processes, single moleculebased synthesis of inorganic nanostructures augments the diffusion and rearrangement processes and lead to single-phase materials. The later aspect is attributed to precursor constitution possessing pre-defined metal-ligand structural units relevant for the constitution of the final nanomaterial. On the other hand, the different compounds present in a reactionmixture randomly collide to form various intermediate species inducing phase separation and element segregation. For instance, a simple mixture of two chemical precursors A and B (Multi Source Precursor, MSP), to produce the binary system AB, may produce a visibly homogeneous mixture but the molecular level scenario can be different and despite a correct global stoichiometry the precursor 'cocktail' may have element segregation and non-ideal $\mathrm{A}: \mathrm{B}$ ratios at the nanometer scale that may be carried forward to the end product (Scheme 2).

As indicated in Scheme 2, the two-component mixture of $\mathrm{Co}(\mathrm{A})$ and $\mathrm{Fe}(\mathrm{B})$ acetates, show multi-step thermal degradation due to individual decomposition profiles of $\mathrm{Co}$ and $\mathrm{Fe}$ precursors as confirmed by the TG/DTA analyses of monometallic acetates. Based on this data, it can be envisaged that the thermal decomposition of such a mixture in high-boiling solvent results in two nucleation and growth processes, which differ on the reaction time 
scales. As a result, the cobalt oxide particles, which are formed $\left(216^{\circ} \mathrm{C}\right)$ first grow to much larger size (ripening), when compared to iron precursors, which decompose later $\left(255{ }^{\circ} \mathrm{C}\right)$ and produces relatively smaller particles.

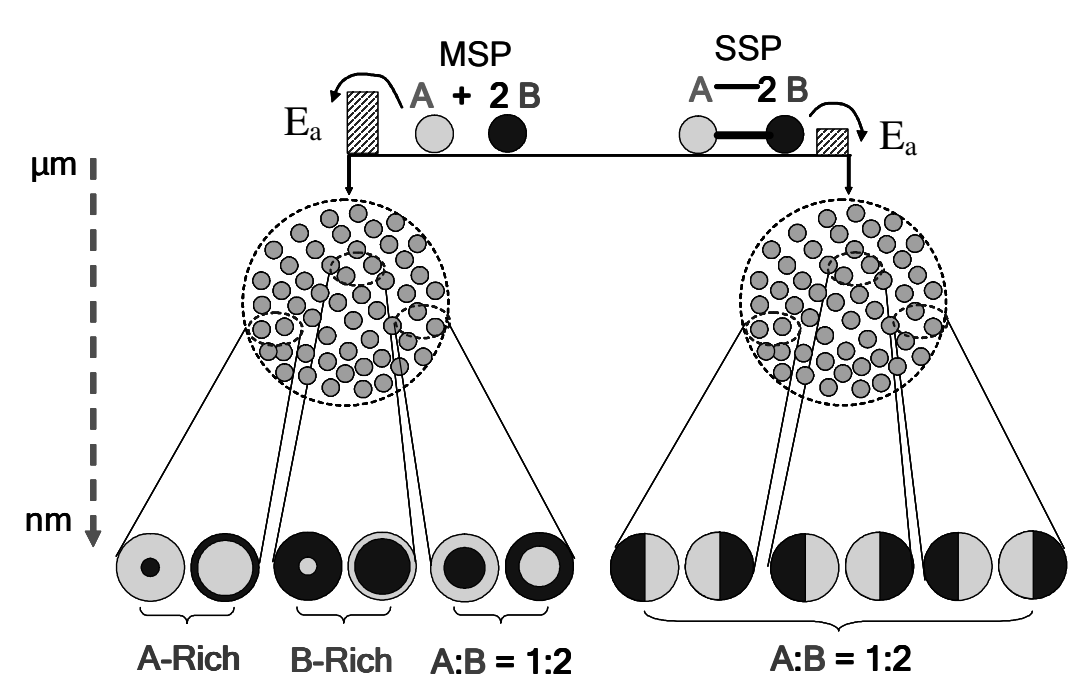

Scheme 2. Chemical nanotechnology: multi-source vs. single source approaches.

\section{Conclusions}

Pure oleic acid - capped cobalt ferrite nanoparticles were prepared by thermal decomposition of a single-source compound $\mathrm{CoFe}_{2}\left(\mathrm{O}^{\mathrm{t}} \mathrm{Bu}\right)_{8}$ in a high boiling solvent. A decomposition temperature of $360-370{ }^{\circ} \mathrm{C}$ was found to be best suited for the synthesis of nanoparticles with around $5 \mathrm{~nm}$ in diameter, that are composed of pure cubic $\mathrm{CoFe}_{2} \mathrm{O}_{4}$ spinell phase, as revealed by XRD and HRTEM analysis. TG-DSC measurements were performed for the single- and mulit-source system and proofed the suitability of the precursor, which decomposes in a single-step process. The obtained particles can be easily dispersed in nonpolar solvents like toluene, hexane or chloroform and exhibit an excellent long-term stability. The variation of the particle size has yet to be improved by variation of the carboxylic chain. In the next step, the nanoparticles should be synthesized in a polar high boiling medium with bi-functional groups to make the particles water-soluble. This should promote the application 
of the material for biological and medical purposes. A study of the magnetic properties of the particles with different capping agents is currently in progress.

\section{Acknowledgements}

We are thankful to the Volkswagen Foundation for financial support of our research.

\section{References}

1. (a) Lee, J. G.; Park, J. Y.; Oh, Y. J.; Kim, C. S. J. Appl. Phys. 1998, 84, 2801. (b) Kodama, T.; Kitayama, Y.; Tsuji, M.; Tamaura, Y. J. Magn. Soc. Jpn. 1996, 20, 305. (c) Cornell, R. M. and Schwertmann, U. The Iron Oxides, VCH Publishers, Weinheim, 2nd ed., 2003.

2. (a) Tiefenauer, L. X.; Tschirky, A.; Kühne, G.; Andres, R. Y. Magn. Res. Im. 1996, 14, 391. (b) Gupta, A. K. and Gupta, M. Biomater. 2005, 26, 3995. (c) Mornet, S. and Vasseur, S. J. Mater. Chem. 2004, 14, 2161. (d) Duguet, E.and Vasseur, S. Nanomedicine, 2006, 1, 157.

3. Cabuil, V.; Neveu, S.; Rosenzweig, R. E. J. Magn. Magn. Mater. 1993, 122, 437.

4. (a) Sun, S. H. and Zeng, H. J. Am. Chem. Soc. 2002, 124, 8204. (b) Si, S. and Kotal, S. A. Chem. Mater. 2004, 16, 3489.

5. (a) Pedro, T. J. Phys. D: Appl. Phys. 2003, 36, 182. (b) Vayssieres, L.; Hagfeldt, A. and Lindquist, S. E. Pure Appl. Chem., 2000, 72, 47.

6. (a) Willis, A. L.; Chen, Z. Y.; He, J.; Zhu, Y.; Turro, N. J. and O'Brien, S. J. Nanomat. 2007, DOI: 10.1155/2007/14858. (b) Rockenberger, J.; Scher, E. C. and Alivisatos, A. P. J. Am. Chem. Soc. 1999, 121, 5608. (c) O'Brien, S.; Brus, L. and Murray, C. B. J. Am. Chem. Soc. 2001, 123, 12085.

7. (a) Klug, K. L.; Dravid, V. P.; Johnson, D. L. J. Mater. Res. 2003, 18, 988. (b) Sun, S. J. Am. Chem. Soc. 2003, 126, 273. (c) Yu, W. W.; Falkner, J. C.; Yavuz, C. T. and Colvin, V. L. Chem. Commun. 2004, 2306.

8. Bronstein, L. M. and Huang, X. L. Chem. Mater. 2007, 19, 3624.

9. Yu, W. W. and Peng, X. Angew. Chem. 2002, 41, 2368.

10. Yin, M. and Chen, Z. Y. J. Mater. Res. 2007, 22, 1987.

11. Meyer, F.; Hempelmann, R.; Mathur, S. and Veith, M. J. Mater. Chem. 1999, 9, 1755.

12. Haas, M. and Mathur, S. unpublished work.

13. Park, J.; An, K.; Hwang, Y.; Park, J. G.; Noh, H. J.; Park, J. Y.; Hwang, J. H. and Hyeon, T. Nature Mater. 2004, 3, 891. 
14. Mathur, S. and Shen, H. "Inorganic Nanomaterials from Molecular Templates" in Encyclopedia of Nanoscience and Nanotechnology ${ }^{\circledR}$ Ed. by Nalwa, H. S. American Scientific Publisher, Vol. 4, p.131, 2004.

\section{Figure Captions}

Figure 1. Processing diagram of $\mathrm{CoFe}_{2} \mathrm{O}_{4}$ nanoparticles from single source precursor $\mathrm{CoFe}_{2}\left(\mathrm{O}^{t} \mathrm{Bu}\right)_{8}$.

Figure 2. (a) TEM and (b) HR-TEM images of cobalt ferrite nanoparticles by single source method.

Figure 3. Powder X-ray diffractogram of $\mathrm{CoFe}_{2} \mathrm{O}_{4}$ nanoparticles obtained by thermal decomposition of $\mathrm{CoFe}_{2}\left(\mathrm{O}^{\mathrm{t}} \mathrm{Bu}\right)_{8}$.

Figure 4. (a) TG curves showing the weight loss characteristic of several precursors upon thermal decomposition. (b) DSC curves for the comparison of single- and multi-source systems recorded with a heating rate of $10 \mathrm{~K} /$ minute under argon atmosphere.

Figure 5. TEM image of cobalt and iron oxide nanoparticles by multi-source method. 
Figure 1

$$
\mathrm{CoCl}_{2}+2 \mathrm{Na}\left[\mathrm{Fe}\left(\mathrm{O}^{\mathrm{t}} \mathrm{Bu}\right)_{4}\right] \longrightarrow \mathrm{Co}\left[\mathrm{Fe}\left(\mathrm{O}^{t} \mathrm{Bu}\right)_{4}\right]_{2}+2 \mathrm{NaCl}
$$
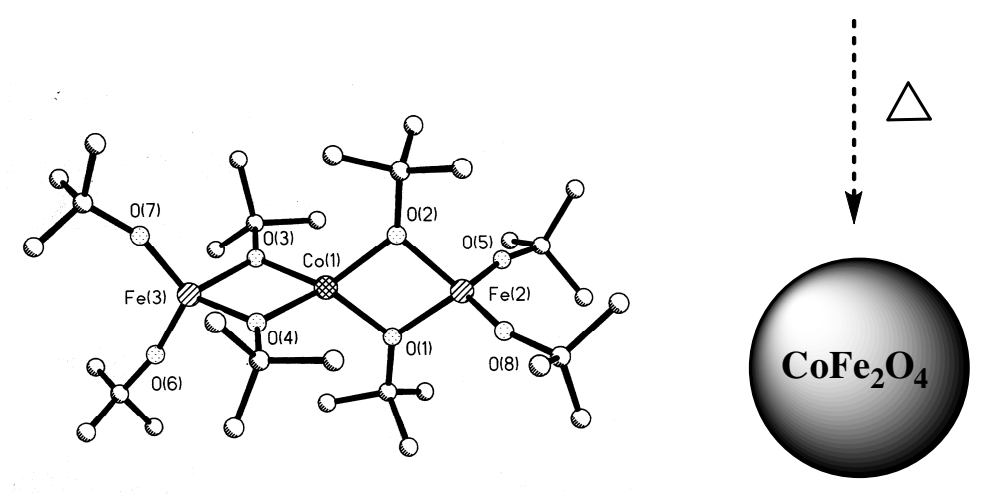
Figure 2a

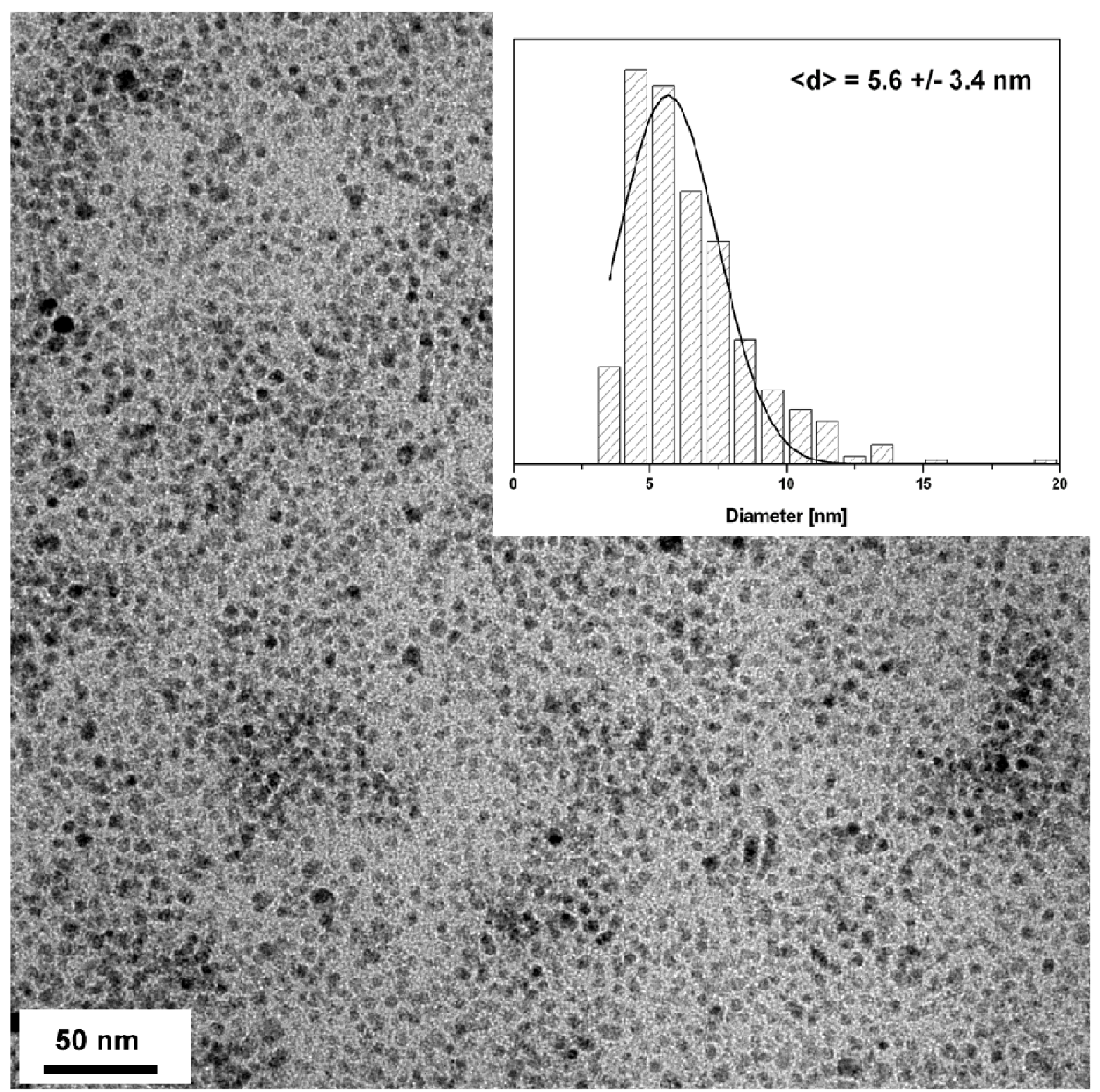


Figure 2b

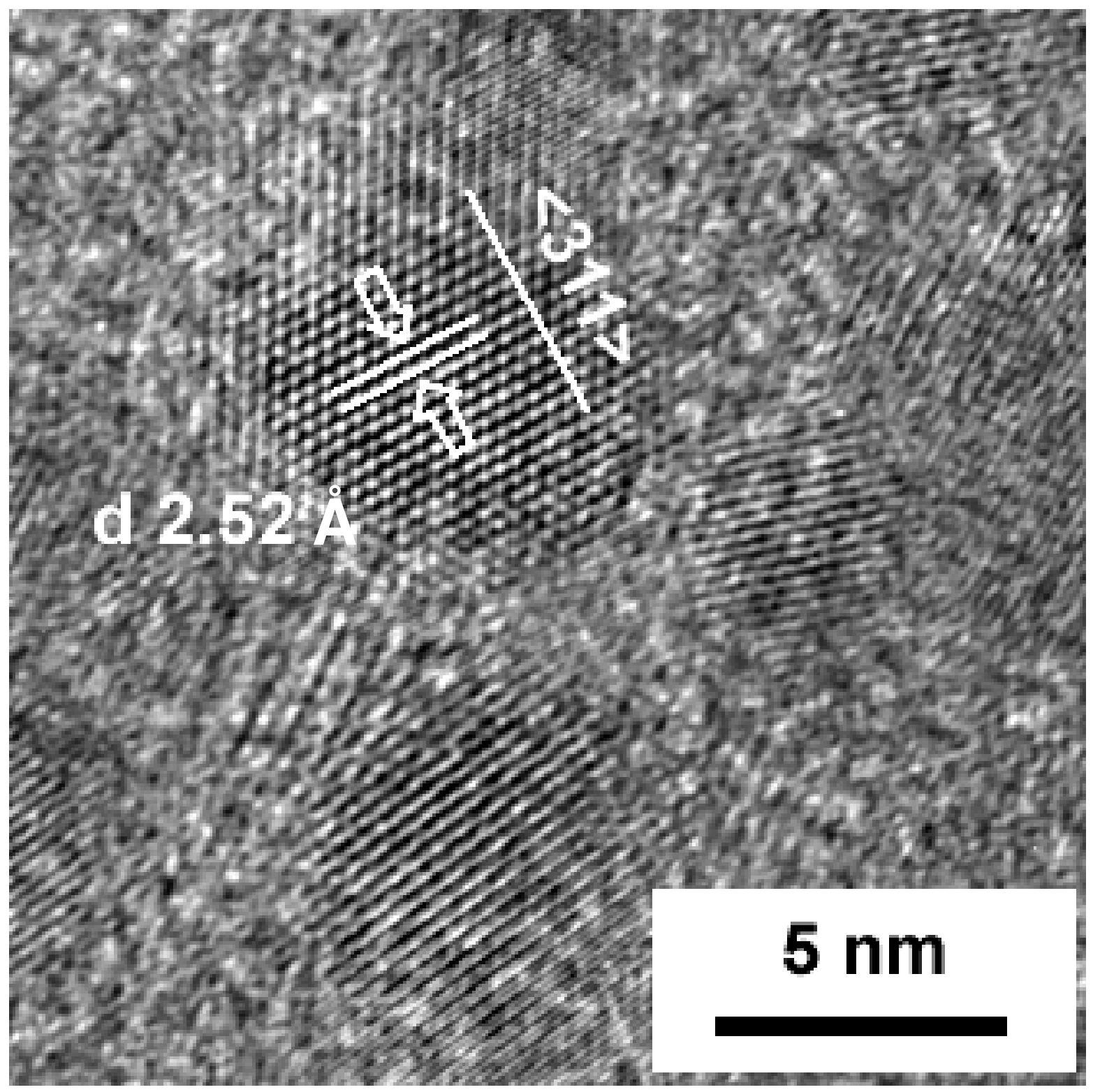


Figure 3

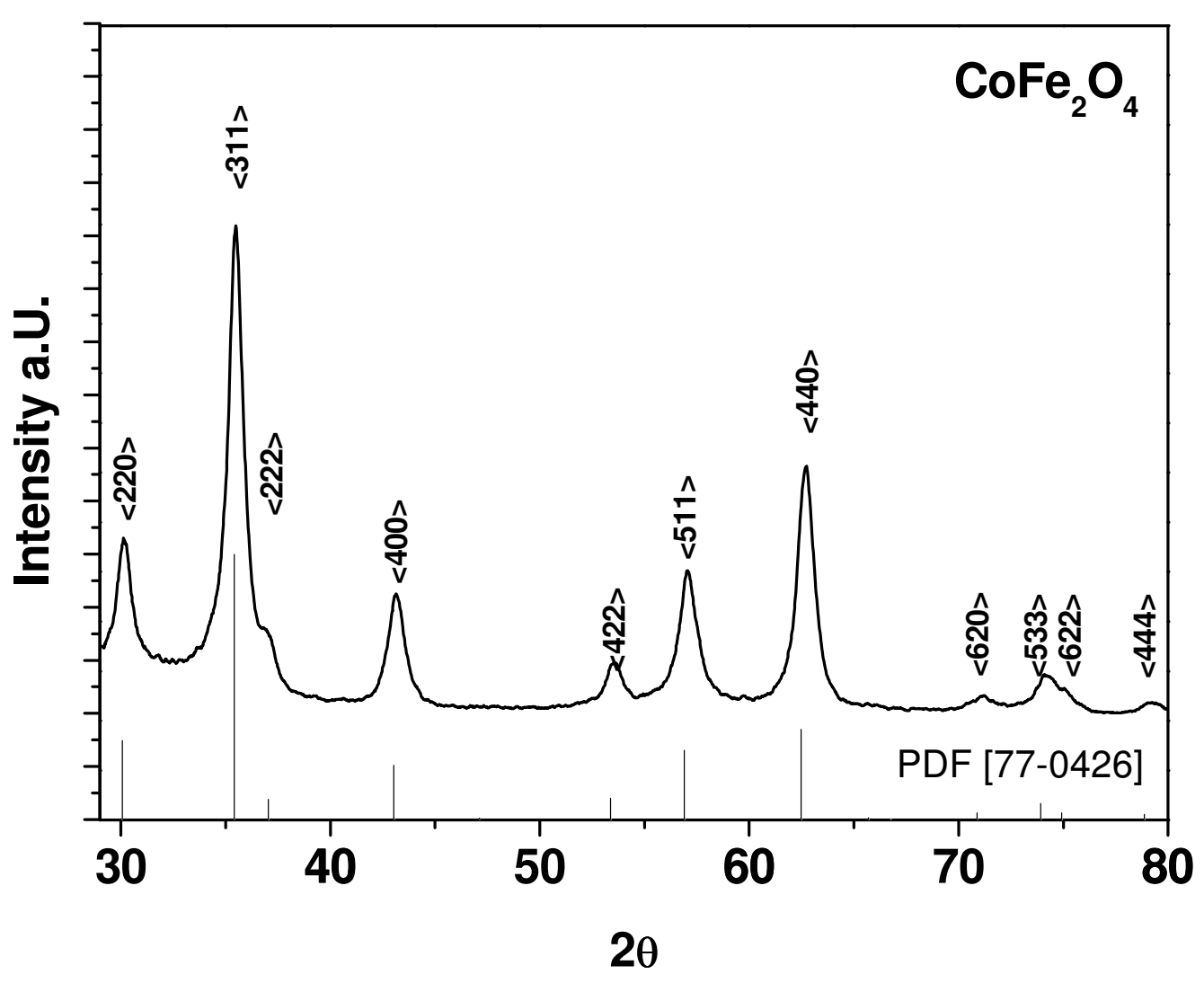


Figure 4a

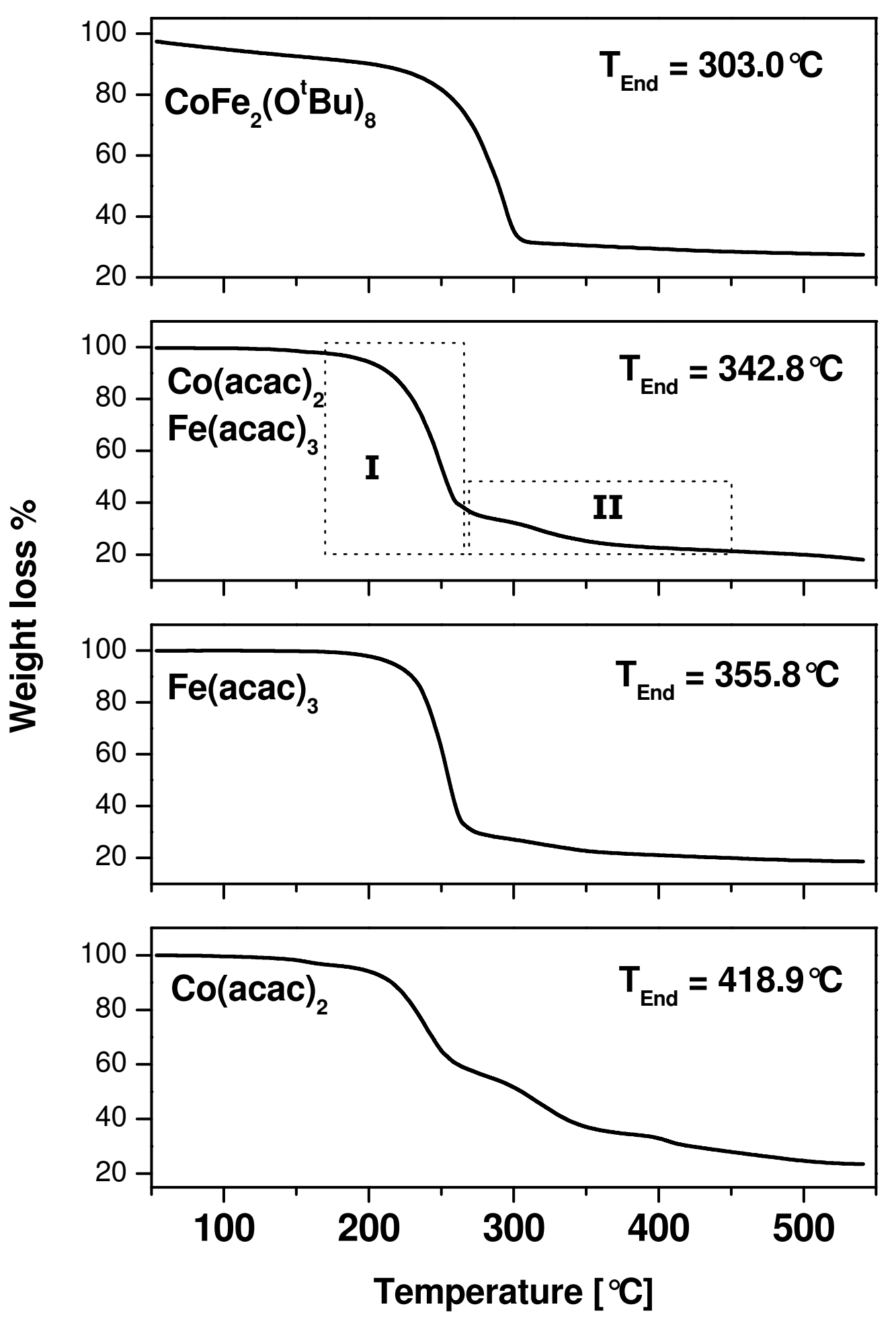


Figure 4b

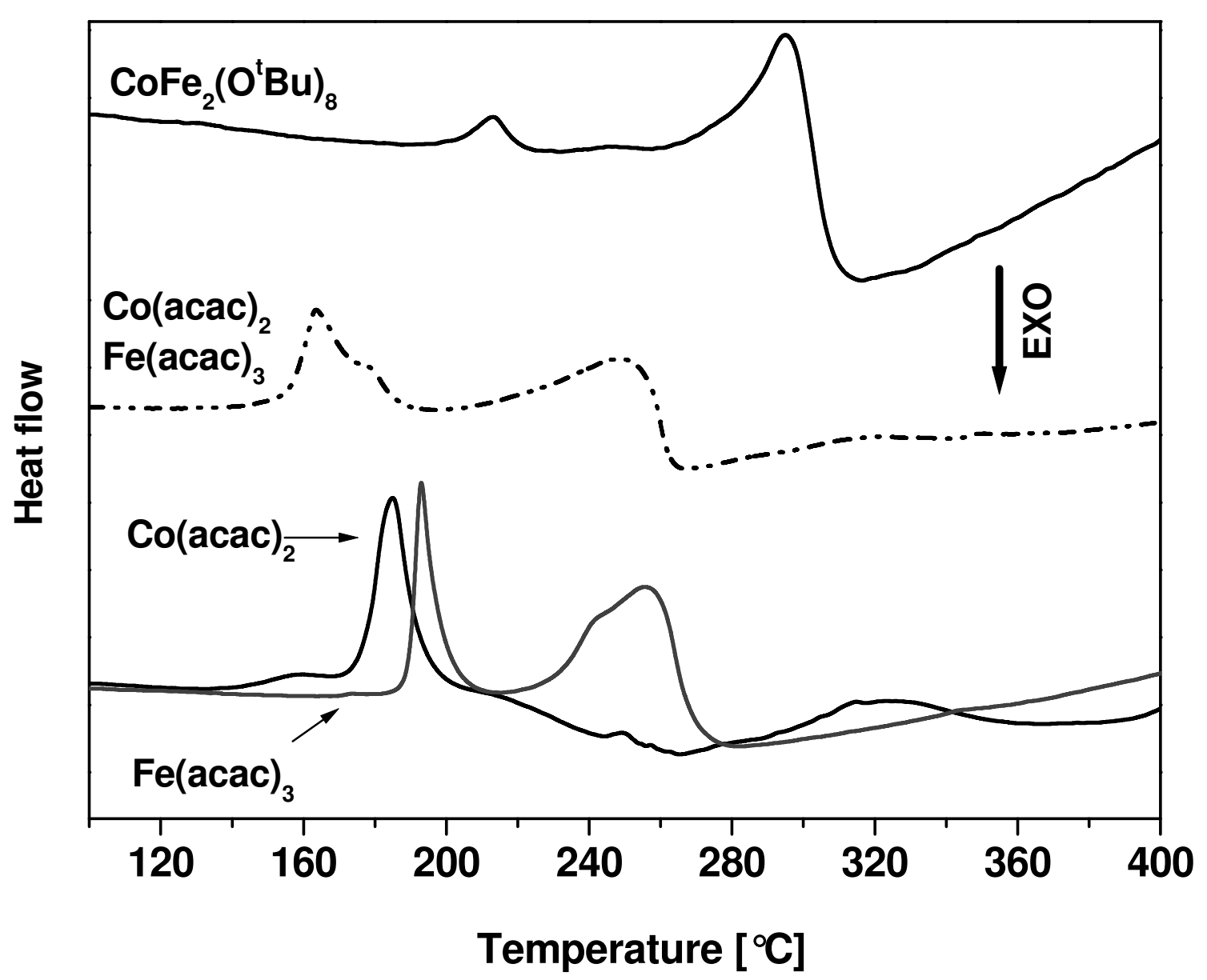


Figure 5

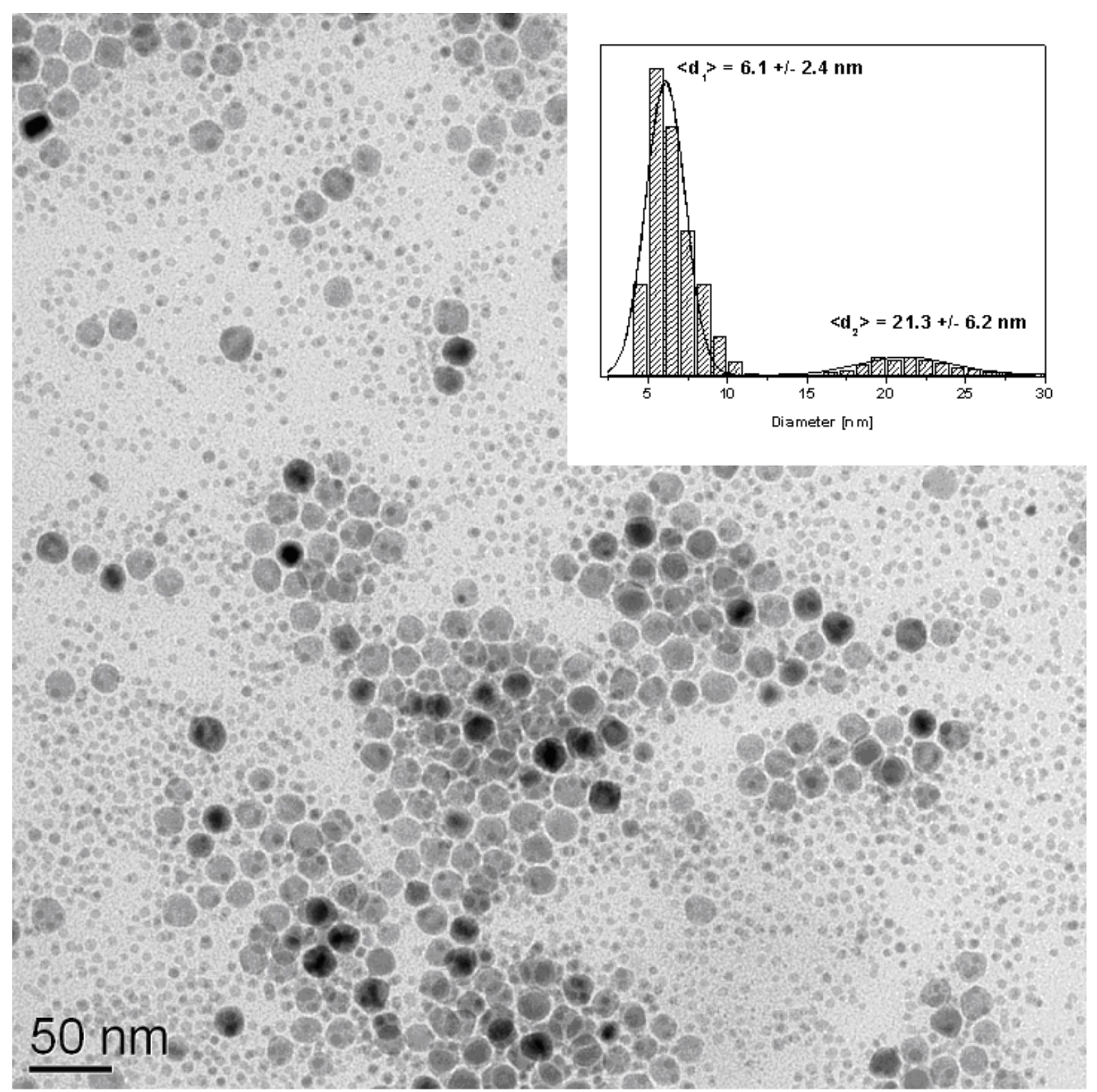

